\title{
Os desafios da organização em rede na atenção psicossocial especializada: o caso do Recife
}

\author{
The challenges of network organization in specialized psychosocial \\ care: the case of Recife
}

Alexciane Priscila Silva', Heloisa Maria Mendonça de Morais' ${ }^{\mathbf{1}}$, Maria do Socorro Veloso de

Albuquerque', Maria Beatriz Lisbôa Guimarães', Tereza Maciel Lyra²

DOI: $10.1590 / 0103-1104202112805$

\begin{abstract}
RESUMO Objetivou-se analisar a atenção especializada em saúde mental da cidade do Recife e seu processo de regulação assistencial. Trata-se de um estudo qualitativo, realizado por meio de análise documental, observação, entrevistas semiestruturadas e diário de campo. Os sujeitos da pesquisa foram gestores e profissionais da Rede de Atenção Psicossocial e da regulação em saúde do Recife. Trabalhou-se com análise de conteúdo do tipo temática, a partir da técnica de condensação de significados. Apesar do histórico de alinhamento com a Reforma Psiquiátrica Brasileira e dos avanços nos processos de desinstitucionalização, a cobertura dos Centros de Atenção Psicossocial/tipo III é insuficiente e há fragilidades de comunicação entre os componentes da rede. Além disso, os ambulatórios de saúde mental atuam em complementaridade ao modelo manicomial. A regulação assistencial é incipiente na saúde mental e centrada no fluxo de marcação de consulta especializada, sem dispositivos de conversação entre os profissionais. Portanto, a atenção na saúde mental com suporte logístico da regulação assistencial se configura como um desafio para a gestão municipal. Por último, ressalta-se que as fragilidades na organização da rede na cidade do Recife podem, ainda, ser agravadas pela agenda antirreformista que vem instituindo mudanças na Política Nacional de Saúde Mental no Brasil.
\end{abstract}

PALAVRAS-CHAVE Regulação e fiscalização em saúde. Saúde mental. Serviços de saúde mental. Assistência à saúde. Serviços comunitários de saúde mental.

ABSTRACT The objective was to analyze the specialized mental health care in the city of Recife and its assistance regulation process. It is a qualitative study, carried out through document analysis, observation, semi-structured interviews and field diary. The research subjects were managers and professionals from the Psychosocial Care Network and health regulation in Recife. Thematic content analysis was performed, based on the condensation of meanings technique. Despite the history of alignment with the Brazilian Psychiatric Reform and the advances in deinstitutionalization processes, the coverage of the Psychosocial Care Centers/ type III is insufficient and there are weaknesses in communication between the components of the network. In addition, mental health clinics are complementary to the asylum model. Assistance regulation is incipient in mental health and centered on the flow of scheduling specialized consultations, without devices for conversation between professionals. Therefore, mental health care, with logistical support for assistance regulation, is a challenge for municipal management. Finally, it is emphasized that the weaknesses in the organization of the network in the city of Recife may, still, be aggravated by the anti-reform agenda that has been instituting changes in the National Mental Health Policy in Brazil.

2 Fundação Oswaldo Cruz (Fiocruz), Centro de Pesquisas Aggeu Magalhães (IAM) - Recife (PE), Brasil.

KEYWORDS Health care coordination and monitoring. Mental health. Mental health services. Delivery of health care. Community mental health services. 


\section{Introdução}

O movimento da Reforma Psiquiátrica Brasileira (RPB) surgiu no final da década de 1970, em um cenário de luta contra a ditadura militar e pela democratização da saúde. No período, o Movimento dos Trabalhadores de Saúde Mental denunciava o descaso e as violências praticadas nos hospitais psiquiátricos, trazendo para o debate a luta social por direitos humanos. Na segunda metade dos anos 1980, com a ampliação da participação social, assumiu-se o lema 'Por uma sociedade sem manicômios', propondo crítica radical ao modelo biomédico em psiquiatria e aos interesses que o sustentavam ${ }^{1}$. Gradualmente, esse movimento induziu a mudanças na política de saúde mental do Estado brasileiro, pelo que foi instituída uma nova legislação com a aprovação da Lei Federal n ${ }^{0} 10.216 / 2001$, que dispõe, entre outras questões, sobre o cuidado preferencialmente em serviços comunitários de saúde mental.

Todavia, a trajetória da RPB apresenta-se marcada por tensões, interesses e forças que disputam modelos e recursos públicos entre uma perspectiva com ênfase na lógica da saúde como direito universal e garantia do cuidado em liberdade e uma outra, privatizante e com reforço à remanicomialização do cuidado ${ }^{2}$.

Apesar de a RPB em curso não se restringir a mudanças nos serviços e tecnologias de cuidado, foi decisiva na reorganização da atenção às pessoas em sofrimento psíquico ao redirecionar a lógica manicomial para o cuidado no território, com ênfase em serviços comunitários ${ }^{3}$. Em 2011, impulsionada pela institucionalização do formato organizativo das Redes de Atenção à Saúde (RAS), a assistência em saúde mental no Sistema Único de Saúde (SUS) passou a ser configurada em Rede de Atenção Psicossocial (Raps) com a promulgação da Portaria n ${ }^{0} 3.080 / 2011^{4}$.

A Raps se organiza em sete componentes - Atenção Básica, Atenção Psicossocial Estratégica, Atenção Hospitalar, Atenção à Urgência e Emergência, Atenção
Residencial Transitória, Estratégias de Desinstitucionalização e Reabilitação Psicossocial -, nos quais estão dispostos serviços como as Unidades de Saúde da Família (USF), os Núcleos de Apoio à Saúde da Família (Nasf), os Centros de Atenção Psicossocial (Caps), os Serviços Residenciais Terapêuticos (SRT), os leitos hospitalares de atenção integral e outros.

Apesar da polissemia acerca do conceito de redes de saúde, a política brasileira define as RAS como:

Arranjos organizativos de ações e serviços de saúde, de diferentes densidades tecnológicas, que integradas por meio de sistemas de apoio técnico, logístico e de gestão, buscam garantir a integralidade do cuidado5(33).

Qualquer que seja a definição adotada, relaciona-se à noção de integralidade, existência de relações horizontais poliárquicas entre os serviços e coordenação do cuidado pela Atenção Primária à Saúde (APS)6.

Considerando a importância da articulação em rede e da organização dos fluxos assistenciais, a regulação assistencial em saúde desponta no Brasil como potente processo que possibilita viabilizar tais fluxos, com a finalidade de promover o acesso equânime e o cuidado integral. Oliveira e Elias ${ }^{7}$ assinalam que apesar de não possuir definição conceitual unificada, a ideia de regulação encontra-se vinculada a noções de sistema e complexidade.

Neste estudo, compreende-se o processo de regulação como instrumento de equilíbrio entre demanda do usuário e prestação de serviços de saúde ofertados por entes públicos ou privados, com objetivo de assegurar o acesso às ações e tecnologias de saúde, de modo a oferecer eficiência ao sistema e equidade à população ${ }^{8}$.

Frente ao exposto, esta pesquisa torna-se relevante ao produzir conhecimento acerca do arranjo organizacional dos serviços de saúde mental estruturados na Raps, ao mesmo tempo que discute a regulação em saúde enquanto dispositivo organizativo dessa rede. Assim, 
tem por objetivo analisar a organização da atenção especializada em saúde mental (Caps e ambulatórios de saúde mental para adultos) na Raps da cidade do Recife e os processos de regulação da atenção que permeiam essa rede.

\section{Metodologia}

Trata-se de estudo descritivo e exploratório, com abordagem qualitativa, do tipo estudo de $\operatorname{caso}^{9}$, ao qual se integrou uma ampla variedade de evidências, tais como documentos, entrevista, observação não participante e diário de campo. A pesquisa teve como espaço de observação a rede de saúde mental da Secretaria Municipal de Saúde (SMS) do Recife, Pernambuco, no ano de 2015.
Em 2015, a Raps do Recife para adultos com sofrimento mental estava distribuída em seis Distritos Sanitários (DS), o que, para esta pesquisa, equivale aos territórios de saúde. Genericamente, cada DS comportava a seguinte configuração de rede: USF, Nasf, policlínicas, nas quais funcionam os ambulatórios de saúde mental, Caps e SRT. Na dimensão macro, somavam-se a essa rede distrital os leitos integrais, distribuídos em dois hospitais filantrópicos pertencentes à rede hospitalar complementar do município. Além disso, a Raps municipal conta com um hospital psiquiátrico público estadual, que se constitui em hospital de referência principalmente para as situações de urgências e emergências psiquiátricas. A referida rede consta na figura 1 . 
Figura 1. Atenção especializada da Raps Recife para adultos em sofrimento ou com transtorno mental por Distrito Sanitário em 2015

6 Residências Terapêuticas

Caps II Esperança

CAPS Caps II Espaço Azul

Policlínica Albert Sabin

(7 psicólogos/4 psiquiatras)

Policlínica Clementino Fraga

(3 psicólogos/2 psiquiatras)
Hospital Evangélico de Pernambuco*

(10 leitos de saúde mental)

Hospital Psiquiátrico Ulysses Pernambucano**

(leitos e emergência psiquiátrica)

6 Residências Terapêuticas

CAPS Caps II José Carlos Souto

Policlínica Amaury Coutinho

(2 psicólogos/1 psiquiatra)

Policlínica Salomão Kelner

(1 psiquiatra)

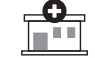

6 Residências Terapêuticas

6 Residências Terapêutic

1 Policlínica Lessa de Andrade

(6 psicólogos/3 psiquiatras)

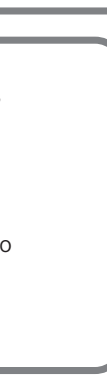
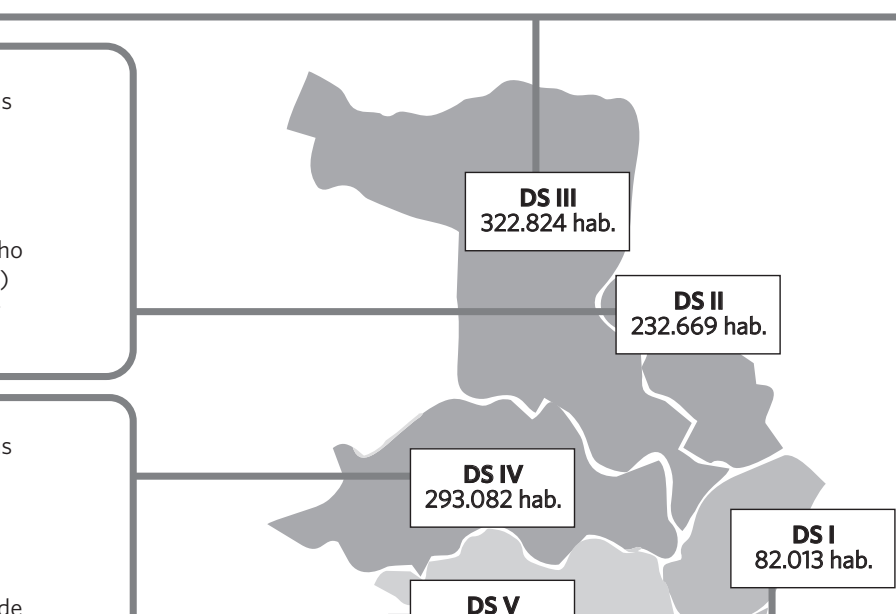

7 Residências Terapêuticas

Caps III Galdino Loreto (24h)

Policlínica Agamenón Magalhães

\section{(6 psicólogos/4 psiquiatras)}

4 Residências Terapêuticas

Caps II Espaço Livremente

Caps III David Capistrno

Policlínica do Pina

Policlínica Armando Marques

(4 psicólogos)

Fonte: Elaboração própria.

*Hospital filantrópico pertencente à rede hospitalar complementar do município.

${ }^{* *}$ Hospital da rede estadual. 
Os dados foram coletados no período de janeiro a maio de 2015, inicialmente, por observação não participante, na qual a pesquisadora realizou uma imersão, no período de janeiro a fevereiro de 2015, acompanhando as reuniões da rede de saúde mental e da Gerência de Saúde Mental Álcool e outras Drogas (GSMAD) enquanto ator externo e na posição de pesquisadora. Na ocasião, foram elaborados diários de campo. Posteriormente, foram realizadas entrevistas com trabalhadores e gestores, cujos critérios de inclusão foram: exercer atividade profissional na Secretaria Executiva de Regulação em Saúde (Sers) ou na Raps do Recife (GSMAD, Caps e DS) e ter idade maior ou igual a 18 anos.
Os participantes da entrevista foram selecionados por meio das seguintes etapas: 1) convite feito no local de atuação do profissional; 2) indicação de profissionais a partir dos entrevistados; e 3) profissionais indicados pelo gestor (quadro 1). As fontes documentais analisadas foram os relatórios de gestão (relatórios anuais de saúde, relatórios de supervisão dos Caps), planos municipais de saúde e planos anuais de saúde, projetos (projeto módulos de reabilitação psicossocial e projeto de fortalecimento da Raps) e atas de reuniões da equipe de desinstitucionalização, todos elaborados no período de 2010 a 2015.

Quadro 1. Demonstrativo do grupo investigado

\begin{tabular}{ll}
\hline Local de atuação & Grupo investigado \\
\hline Sers & 4 trabalhadores Sers \\
Raps Recife & 2 gestores da GSMAD \\
& 2 trabalhadores dos Caps \\
& 2 gestores - 'referência distrital em saúde mental'* \\
\hline
\end{tabular}

Fonte: Elaboração própria.

*São responsáveis pela condução da Política Municipal de Saúde Mental em uma unidade territorial delimitada, com a atribuição de ser elo entre a GSMAD e a rede de serviços de saúde dessa unidade que corresponde a um Distrito Sanitário.

As entrevistas semiestruturadas foram gravadas, transcritas e seguiram roteiro prévio contendo as seguintes questões: identificação das prioridades e dos objetivos da política municipal de saúde mental; organização e estrutura da Raps - Recife, para adultos com sofrimento mental; e a interface entre regulação em saúde e saúde mental. Cada entrevistado recebeu uma codificação (trabalhador ou gestor - número arábico - localização institucional Raps Recife ou Sers). Das falas dos entrevistados emergiram temas como papel do Caps na Raps, interlocução entre atenção especializada e APS, e as reverberações manicomiais dos ambulatórios de saúde mental.

As entrevistas e os documentos foram analisados mediante análise de conteúdo temática, a partir da técnica de condensação de significado $^{10}$. Assim, adotaram-se as seguintes etapas: leitura cuidadosa para apreensão do sentido do todo; identificação das 'unidades naturais'; definição dos temas centrais extraídos a partir da compreensão acerca das unidades naturais; interrogação quanto à relação entre as ideias centrais e os objetivos propostos pelo estudo; e descrição sintética dos temas identificados conforme a interpretação do pesquisador acerca dos objetivos da pesquisa.

A pesquisa foi aprovada pelo Comitê de Ética em Pesquisa da Universidade Federal de Pernambuco (parecer $n^{\circ} 818.711 / 2014$ ), e foram respeitados os aspectos éticos conforme Resolução no 466/ 2012, do Conselho Nacional de Saúde/Ministério da Saúde. 


\section{Resultados e discussão}

Os resultados a seguir estão organizados em três temáticas, que emergiram a partir do corpus do estudo. Foram elas: o lugar dos Caps na Raps do Recife; a interlocução entre os componentes da Raps (Caps, as unidades de APS e os ambulatórios de saúde mental); e a regulação assistencial na saúde mental.

\section{O lugar dos Caps na Raps na cidade do Recife}

A conformação atual da Raps local reflete décadas de construção de uma rede assistencial com propósito de operar no modo psicossocial, todavia, essa conformação se deu no contexto desafiador de desmontagem do aparato manicomial de um dos maiores polos hospitalocêntricos com características asilares e excludentes do País. Até 2001, havia sob gestão municipal sete hospitais psiquiátricos, que correspondiam a $60,2 \%$ do total de leitos do município"1.

A partir dessa época, Recife promoveu um processo de desinstitucionalização e implantação de serviços extra-hospitalares impulsionado pelo contexto de mudança de gestão para uma gestão popular, pela aglutinação de atores-chave vinculados à luta pela RPB à gestão municipal e devido à municipalização de serviços estaduais de saúde mental ${ }^{12}$.

Assim, o município implantou uma rede regionalizada de serviços substitutivos na qual atualmente existem 17 Caps. Em 2015, a cidade do Recife possuía uma cobertura de 1,2 por 100.000 habitantes, o que indicava uma 'cobertura muito boa' segundo os parâmetros do Ministério da Saúde ${ }^{13}$. Contudo, estudo desenvolvido em $2010^{14}$ analisou os indicadores de cobertura por região geográfica e demonstrou que o uso desse indicador de cobertura global é falacioso, na medida em que não representa as realidades regionais e mascara as regiões deficientes.

Ressalta-se que, para além da expansão da rede extra-hospitalar, há evidências de que o município incorporou o território enquanto componente central do trabalho para o processo de desconstrução da cultura manicomial, particularmente, com a implantação de mecanismos de desinstitucionalização como as residências terapêuticas ${ }^{11}$ :

[...] eu nunca tinha supervisionado as SRTs, e aí eu me deparei com usuários, com moradores, que, antigamente, eles tinham sido meus usuários em uma determinada instituição (psiquiátrica), há anos [...] E, hoje... quando eu cheguei na casa, eu realmente me surpreendi, me emocionei realmente de vê-lo com o resgate da cidadania, a liberdade dentro da própria comunidade, o poder estar partiIhando, interagindo dentro da casa. (Trabalhador 01 - Sers).

Quanto à tipologia dos Caps, existem cinco modalidades, a saber: Caps I, II e III (com funcionamento 24 horas), Caps i (infanto-juvenil) e Caps ad (álcool e outras drogas), os quais se diferenciam conforme o porte, a ordem crescente de complexidade e segundo a clientela atendida. Entre os 17 Caps da rede do Recife, oito deles são da modalidade adulto transtorno, sendo que apenas dois funcionavam durante as 24 horas $^{15}$.

Considerando que quase todos os territórios de saúde da cidade do Recife possuem mais de 150.000 habitantes ${ }^{16}$, a cidade encontrava-se em desacordo com a normativa nacional ${ }^{4}$, que preconiza a existência de Caps tipo III para territórios com esse quantitativo populacional. Destaca-se que outros municípios também possuem uma cobertura insuficiente de serviços Caps III, sugerindo dificuldades assistenciais na atenção à crise das pessoas em sofrimento psíquico ${ }^{3}$.

Ademais, os Caps do tipo II foram apontados pelos entrevistados como insuficientes para atender à demanda do território ao qual são referenciados. Afirmam, também, que a escassez de Caps III repercute em sobrecarga de trabalho e fragmentação da atenção, levando esses serviços, por vezes, a atuarem sob a lógica do 'apagar fogo', conforme mencionado por um dos entrevistados. 
Onocko-Campos e colaboradores ${ }^{3}$ sinalizam o risco de retorno às práticas segregadoras que ocorriam em instituições psiquiátricas quando há uma insuficiência na disponibilidade de Caps. Além disso, a falta de recursos humanos e financeiros adequados às necessidades dos Caps interfere no processo de trabalho desses serviços ${ }^{17}$. E, no município, apesar da ampliação da rede extra-hospitalar e do fechamento dos leitos dos hospitais psiquiátricos, os recursos oriundos do fechamento desses leitos não foram revertidos para a rede psicossocial ${ }^{11}$.

Apesar da limitação de cobertura de Caps III, os sujeitos entrevistados destacam a importância do dispositivo Caps e o compreendem como estratégico para consolidar a saúde mental nos territórios, particularmente, devido ao movimento que provoca no agenciamento de redes intersetoriais e de encontros dos sujeitos. Ademais, as avaliações sobre os serviços substitutivos indicam avanços na garantia de cuidado humanizado, participativo e comunitário ${ }^{18}$.

No modelo inicial de superação das práticas manicomiais, o Caps, enquanto dispositivo estratégico, ocupava uma posição centralizada no desenho de rede e tinha como atributos organizar, regular e articular os dispositivos do território sob sua responsabilidade, tais como unidades de saúde e outras redes intersetoriais ${ }^{19}$. Com a mudança no desenho organizativo no formato de Raps, o Caps mantém sua característica estratégica de articulador, mas a ordenação do cuidado passa a ser compartilhada com a APS 4 , uma vez que esta se configura em centro de comunicação e ordenação de cuidado e assume a centralidade no desenho organizativo ${ }^{20}$.

Para os trabalhadores entrevistados neste estudo, essas duas configurações de desenhos de redes coexistem e reverberam divergências sobre o papel do Caps na Raps.

[...] o Caps deveria ser o centro de saúde mental, o centro dessa rede. O Caps é um modelo substitutivo ao modelo hospitalocêntrico, então, teoricamente, emergência, urgência e tudo, a gente deveria dar conta. (Trabalhador 07 - Raps Recife).
O Caps é mais um equipamento de saúde, dentro dessa rede, tão importante quanto o Nasf, o consultório na rua, a atenção básica, as unidades da assistência social. (Trabalhador 10 - Raps Recife).

Faz-se necessário diferenciar a função de organizar e articular a rede de saúde mental de um determinado território em contraste com assumir a centralidade no desenho dessa rede. Ao presumir que o papel do Caps seria o de 'dar conta' de todas as dimensões de cuidado, corre-se o risco de condicionar o avanço da RPB a uma resolutividade utópica desse serviço. A compreensão de que ser estratégico não significa acolher a totalidade das questões que envolvem a saúde mental, mas relaciona-se com o articular e tecer redes ${ }^{21}$, é imprescindível para se pensar o trabalho compartilhado inter e intrasetorial e, assim, avançar no desafio de garantir a reinserção social e o respeito às subjetividades.

Além disso, ao incorporar a atenção psicossocial como premissa do cuidado em saúde, ampliaram-se as possibilidades de atuação na saúde mental. Nesse sentido, as intervenções em saúde mental não se encerram no indivíduo e nas medidas curativas, mas avançam para a produção de práticas que trazem à cena os sujeitos em sofrimento psíquico no cotidiano de seus territórios. Destaca-se que a noção de território incorporada nesta pesquisa diz de sua dimensão simbólica e cultural, que denota um caráter itinerante e vivo, constituído a partir da narrativa pessoal dos sujeitos e consoante a acepção de territórios existenciais de Deleuze e Guattari22 . Assim, a APS se constitui como recurso a ser explorado no que diz respeito ao cuidado em saúde mental ${ }^{23}$, uma vez que a atuação nesse campo não se restringe aos consultórios e aos espaços especializados, mas refere-se, também, ao estabelecimento de vínculos, relação de contrato e corresponsabilidade entre profissionais e comunidade.

Cabe lembrar que o Caps é um serviço comunitário que se caracteriza por ser porta aberta, isto é, um dispositivo que acolhe, prioritariamente, pessoas com transtornos mentais 
graves e persistentes em situações de crise ou que necessitem de reabilitação psicossocial. Porém, a APS, pelos atributos que a caracterizam ${ }^{\mathbf{2 4}}$, apresenta-se como a instância mais adequada para assumir a responsabilidade pelo percurso terapêutico dos usuários na maioria das situações de adoecimento, inclusive naquelas de sofrimento mental.

Assim, concomitante à reorganização da rede de saúde mental no formato de Raps, reafirma-se o papel do Caps enquanto dispositivo estratégico para atenção à crise e reinserção social, mas sob a radicalidade de uma configuração de rede poliárquica com funcionamento circular, na qual a APS é o centro de comunicação e de ordenação do cuidado.

\section{Os desafios da interlocução entre os componentes da Raps}

A cidade do Recife possuía, em 2015, uma cobertura da Estratégia Saúde da Família (ESF) de aproximadamente $53 \%{ }^{25}$, o que implica extensas áreas descobertas. Nas situações em que não há USF de referência no território, o Caps articula ações com outros dispositivos, como os da assistência social - a exemplo dos Centros de Referência de Assistência Social -, as equipes do Nasf e outros serviços da APS, como as unidades básicas tradicionais.

Falhas na interlocução entre Caps e UBS foram apontadas pelos trabalhadores de saúde mental entrevistados como questões que comprometem o funcionamento dos Caps e repercutem no acompanhamento dos sujeitos, como a permanência dos usuários nos Caps por longos períodos, o reforço do ciclo de retorno ao Caps por fragilidade na rede de cuidado territorial e os encaminhamentos pouco qualificados, gerando sobrecarga aos profissionais.

Um dispositivo que poderia melhorar a articulação entre o Caps e as UBS seria o Apoio Matricial (AM). Ressalta-se que o AM é sugerido como a principal estratégia para qualificar e ampliar a resolutividade das ações da APS ${ }^{26}$, na medida em que pode viabilizar a articulação de redes de cuidado com ênfase na corresponsabilização dos atores envolvidos, potencializar novas abordagens em saúde mental, ordenar e direcionar os encaminhamentos para a rede especializada, entre outras possibilidades.

No entanto, de acordo com alguns dos entrevistados, a atuação do AM vem se dando de modo pontual e não sistemático pela insuficiência de profissionais, pela superlotação dos Caps e por questões de logística, como, por exemplo, a baixa disponibilidade de transporte. Esse achado corrobora a literatura, uma vez que os principais desafios à efetivação desse dispositivo dizem respeito a questões estruturais como sobrecarga dos profissionais, superlotação dos serviços, cobrança por produtividade, entre outros ${ }^{27}$.

Todavia, no percurso histórico do município, particularmente na gestão 2001-2004, houve uma aposta nas ações de saúde mental na APS, com formação de 'equipes de retaguarda', as quais tinham a função de impulsionar a ação territorial dos Caps por meio do processo no qual as equipes de generalistas das USF teriam o apoio/retaguarda de equipes de saúde mental 28 .

Além disso, essas discussões resultavam em articulações sobre os fluxos assistenciais no território. Todavia, essa proposta foi se desarticulando ao longo dos anos, deixando questionamentos quanto à cisão criada entre os profissionais que atuavam no território e os demais membros da equipe que permaneciam dentro dos Caps. E, em 2009, a gestão abandonou essa organização, visto que nacionalmente estava posto o modelo do Nasf $\mathbf{2 8}$.

Em estudo 29 que avalia a implantação do apoio matricial dos Caps ad do Recife, verifica-se que o apoio matricial se encontra parcialmente implantado no município com diferentes graus de implantação por DS e que os trabalhadores das USF e dos Caps o identificam como estratégia de articulação e suporte para a consolidação da rede de assistência.

Deveras, um dos desafios da RPB é a inserção da saúde mental na APS, principalmente por meio da $\mathrm{ESF}^{30}$. Destaca-se que os 
profissionais da APS estão inseridos em um contexto de hegemonia biomédica, que resulta em uma lógica de cuidado em saúde mental limitada à terapêutica medicamentosa e a encaminhamentos aos especialistas. Ademais, comumente, é relatado por esses profissionais o sentimento de despreparo, insegurança e receio de lidarem com as situações complexas do sofrimento psíquico ${ }^{26,31}$.

Pela Portaria $n^{0} 3088 / 2011$, os ambulatórios em saúde mental não eram previstos enquanto dispositivos de cuidado, aparecendo somente na Portaria ${ }^{\circ}$ 3588/2017 como componentes da Raps. Vale salientar que as práticas ambulatoriais possuem larga tradição no cuidado em saúde mental, mas caminham embrigadas no modelo da psiquiatria comunitária e preventiva americano, que, apesar de identificar o ambulatório como um espaço de tratamento alternativo ao asilo, não produziu uma ruptura com o modelo biomédico e com a psiquiatria tradicional. Para Severo e Dimenstein ${ }^{32}$, o processo de ambulatorização da saúde mental posicionou esses serviços entre dois modelos antagônicos: um que reforça o modelo médico-centrado e outro com características condizentes com a prática de atenção psicossocial.

No Recife, a implantação dos primeiros ambulatórios ocorreu dentro dos hospitais psiquiátricos, e esse serviço se configura como o primeiro embrião do redirecionamento da assistência. Todavia, o seu processo de ampliação, particularmente na década de 1980, refere-se à diminuição dos custos hospitalares, à inclusão de novas categorias profissionais na rede pública (psicólogo e assistente social), sem que a lógica inerente ao modelo manicomial fosse alterada ${ }^{\mathbf{2 8}}$.

Neste estudo, o ambulatório foi identificado pelos entrevistados com características de ser um espaço de cuidado intermediário entre a prevenção e a crise, ou seja, entre a UBS e o Caps:

[...] o ambulatório, não está na Raps, mas não podemos vislumbrar nossa rede sem ele. Ainda não temos uma atenção básica que dê conta dessas pessoas sem o ambulatório [...] e ele precisa ser cuidado para que faça sua função, que, nesse momento, é de aproximar os profissionais da atenção básica do lugar que vai ser deles nesse modelo que hoje o Ministério da Saúde preconiza. (Gestor 09 - Raps Recife).

Damous e Erlich ${ }^{33}$, ao contextualizarem o papel do ambulatório na Raps, particularmente no que concerne ao campo da psicologia, circunscrevem esse dispositivo no nível de atenção secundária com função complementar à atenção primária e aos Caps, e o caracterizam como peculiar à rede por ofertar cuidado especializado de modo continuado, tendo como principal modalidade de acompanhamento a psicoterapia - modalidade que o caracteriza e particulariza entre os demais serviços.

Além disso, segundo as autoras, apesar dos Caps também ofertarem cuidado especializado, distinguem-se dos ambulatórios pelas suas demandas. A clientela do ambulatório é aquela que tem certo grau de autonomia e suporta aguardar, mas que necessita de um trabalho de escuta e fala continuada que deve ser conduzido pelo especialista. Portanto, os ambulatórios se destinam a uma clientela que pode transitar entre a UBS e o Caps.

Apesar de ganharem espaço na assistência em saúde mental, os questionamentos acerca da ineficácia dos ambulatórios e do quanto podem se prestar à reafirmação do modelo tradicional se intensificaram a partir dos anos de 1990, em razão de promoverem a cronificação da clientela, induzirem à fármaco-dependência e à ambulatorização da psiquiatria ${ }^{33}$.

Nessa direção, os entrevistados ressaltam que a prática de cuidado nos ambulatórios é enraizada na sintomatologia e na medicalização, consequentemente, os usuários permanecem por anos recebendo prescrições de psicotrópicos, sem se considerarem as estratégias de promoção de saúde.

Ter cinco psiquiatras no ambulatório vai resolver? Acredito que não, se continuar no mesmo modelo de acompanhar um indivíduo por dez anos tomando Clonazepan à noite [...] Ao invés 
do indivíduo desenvolver a dependência, ele poderia estar no clube dançando [...] e ser acompanhado pelo clínico na atenção básica. E a cada três meses, [...] esse colega clínico poderia se comunicar com o Caps para fazer uma avaliação. (Trabalhador 10 - Raps Recife).

Consoante com esse resultado, estudos realizados em ambulatórios de saúde mental na região Sul do Brasil mostram que praticamente todos os encaminhados à consulta psiquiátrica recebem prescrição de psicofármacos e que há uma baixa frequência de alta ambulatorial dos casos encaminhados à psiquiatria ${ }^{34}$, com atendimentos que privilegiam consultas individuais centradas no modelo curativo ${ }^{35}$.

A lógica de atendimentos exclusivamente individuais, centrados na remissão de sintomas e na medicalização, em detrimento de práticas mais participativas e grupais, confirma a persistência do modelo psiquiátrico tradicional, que, como demonstram os resultados desta pesquisa, é uma característica do funcionamento dos ambulatórios da cidade do Recife. De acordo com as falas dos entrevistados, os usuários atendidos nesses serviços permanecem por anos em atendimento psiquiátrico com a finalidade exclusiva de manter a prescrição medicamentosa, particularmente de ansiolíticos. São frequentes as longas filas de espera devido à pouca rotatividade de usuários, ademais, atribui-se a esse dispositivo uma indicação generalista de espaço para acolhimento dos usuários egressos dos Caps por alta clínica, questões que reforçam práticas tutelares e cronificantes. Tais resultados indicam a necessidade de se rever esse tipo de oferta de serviços ambulatoriais, alinhando-os com a proposta da RPB.

Alguns autores, como Santos, Oliveira e Yamamoto ${ }^{36}$, identificam um conjunto de problemas - a carência de profissionais, os problemas de acessibilidade e a desarticulação com a rede de saúde - que perpassa o cotidiano da assistência ambulatorial, problemas esses igualmente identificados na realidade da rede ambulatorial de saúde mental na cidade do Recife.
O modo de operar dos ambulatórios do Recife sobrevive com contornos que expõem reverberações manicomiais, e, como afirma $\mathrm{Melo}^{\mathbf{2 8}}$, a gestão municipal ainda não assumiu uma posição de extinção desses serviços ou de reestruturação na perspectiva da atenção psicossocial.

\section{Regulação assistencial na saúde mental}

A partir da implantação da Política de Saúde Mental na cidade do Recife, foram introduzidas algumas ações de regulação da atenção, tais como supervisão hospitalar, credenciamento de serviços e acompanhamento contábil e financeiro. Foi, entretanto, a partir da instituição da Raps que surgiu a discussão sobre fluxo assistencial na rede de saúde mental.

Na organização dos fluxos assistenciais, observa-se haver distinção entre o acesso às consultas psiquiátricas e às psicológicas. As consultas psiquiátricas eram reguladas via Sistema Nacional de Regulação (Sisreg), e as consultas psicológicas eram marcadas nas policlínicas por demanda espontânea ou através de encaminhamento direto dos Caps ou das UBS - apenas em duas unidades o primeiro acesso é regulado via Sisreg, mas as consultas de retorno são marcadas de acordo com a agenda do profissional.

Para alguns entrevistados, a não regulação das consultas de psicologia possibilita aos profissionais do Caps maior facilidade para agendá-las, visto que as consultas são marcadas por telefonema entre os profissionais. No entanto, a informalização desse fluxo condiciona o acesso do usuário a relações pessoais mantidas entre os profissionais da rede, o que pode ocorrer satisfatoriamente ou não. $O$ contato informal entre profissionais para agendamento de consultas consiste em uma realidade também de outros municípios $\mathbf{2 4 , 3 6}$, todavia, ocasiona acesso desigual aos pontos de atenção e geralmente não segue orientações clínicas.

As falas dos entrevistados sugerem que a necessidade de regular o fluxo assistencial 
nesses ambulatórios, particularmente nos de psiquiatria, ocorre devido ao contexto de resistência dos profissionais em avançar no processo de alta clínica, o que ocasiona uma sobrecarga para esses serviços, repercutindo na ausência de agenda para novas consultas e na manutenção da fila de espera. Assim, o principal objetivo desse processo é equacionar o problema da demanda reprimida.

Os entrevistados mencionaram, ainda, que uma das formas de enfrentamento dessa demanda reprimida deveria ser a realização de AM entre os psiquiatras do ambulatório e os médicos das UBS, no sentido de qualificar o encaminhamento para a rede de especialistas. Todavia, destoando dessa compreensão, que coloca o AM como estratégia para enfrentamento da demanda reprimida, concorda-se com Gryschek e Pinto ${ }^{26}$, segundo os quais o AM, por meio da educação permanente, qualifica as equipes e promove assistência conjunta e compartilhada, atuando como um dispositivo de consolidação da saúde mental na APS.

Foi possível observar, portanto, que a regulação em saúde mental no município ainda permanece centralizada no fluxo de marcação de consulta especializada, na qual a perspectiva que se sobrepõe é a da psiquiatria tradicional, em detrimento de uma perspectiva de rede que contemple, além da discussão de fluxos e ofertas de consultas, a comunicação sistemática entre os especialistas e generalistas e a coordenação do cuidado pela APS. Sousa e colaboradores ${ }^{37}$ pontuam que a fragmentação do cuidado e o acesso restrito à assistência especializada são realidades não superadas na rede assistencial do Recife, apesar dos fluxos assistenciais estabelecidos via Sisreg.

Nesse sentido, alguns arranjos são apresentados na literatura ${ }^{38} \mathrm{e}$ indicam a construção de um formato de atenção especializada coerente com a Raps, na qual o acesso à atenção especializada se organiza a partir do AM, seja por meio das experiências dos Nasf ou dos Caps, propiciando regulação negociada dos casos entre a USF e o especialista em saúde mental, reduzindo a lista de encaminhamento.
Para Chazan e colaboradores ${ }^{39}$, as relações de saber mais horizontais trazem avanços nas práticas e na formação para as equipes da ESF e equipes dos Nasf enquanto apoio matricial, embora ainda haja desafios para garantia da efetividade de suas ações, que podem ser intensificados com a aprovação da nova Política Nacional de Atenção Básica (PNAB), devido às mudanças e restrições ao trabalho e financiamento dessas equipes.

Destaca-se que desponta como dificuldade nesse processo de regulação a falta de legislação específica que estabeleça parâmetro assistencial. A Sers utilizava como parâmetro a Portaria $\mathrm{n}^{0} 1.101$, de 2002 - revogada pela Portaria $\mathrm{n}^{\mathrm{o}}$ 1.631 , de $1^{\circ}$ de outubro de 2015 , que aprova critérios e parâmetros para o planejamento e a programação de ações e serviços de saúde no âmbito do SUS - porém, não contemplava os atendimentos psicológicos e, para os participantes desta pesquisa, não correspondia aos desafios atuais da cobertura assistencial do SUS. Além do mais, o acompanhamento através da psicoterapia, devido ao seu caráter contínuo, singular e subjetivo, não permite precisar ou fixar um número de consultas para que o processo de cuidado dos sujeitos esteja concluído.

Dessa forma, impõe-se o desafio à SMS do Recife de aprofundar a interface entre regulação e saúde mental para além da construção coletiva de protocolos assistenciais e fluxogramas. Embora sejam necessários, cabe à SMS conformar a Raps municipal e sua regulação. Nesse sentido, concorda-se com Baduy e colaboradores ${ }^{40}$ que a regulação precisa ter mecanismos que possibilitem encontros, redes de conversações da política e da clínica, uma vez que é nesses espaços que acontece a produção do trabalho e do cuidado integral em saúde, para evitar que o processo de regulação assistencial se configure em barreira de acesso.

\section{Considerações finais}

Este estudo mostrou que a conformação atual da Raps local remete a décadas de construção 
de uma rede assistencial com ênfase no seu caráter comunitário consoante com o modo psicossocial. Todavia, a atenção especializada dessa rede apresenta algumas fragilidades, como o insuficiente número de Caps III e as dificuldades de articulação em rede para atenção à crise, sendo imperativo que os Caps tipo II se qualifiquem para a modalidade III e que se estabeleça apoio técnico de serviços de urgência bem articulados com o conjunto da rede.

Além disso, os ambulatórios de saúde mental assumem a posição de complementaridade ao modelo manicomial, na medida em que conferem centralidade ao saber psiquiátrico, à medicalização e à atenção individualizada, em dissonância com a RPB.

Ainda, a regulação assistencial da Raps na cidade do Recife é incipiente, centraliza-se nos fluxos e na oferta de consultas especializadas. Não tem contemplado uma comunicação sistemática entre os especialistas e generalistas e se apoiado na coordenação do cuidado pela APS. Além disso, novos parâmetros assistenciais deverão ser estabelecidos, bem como a conformação de espaços de regulação negociada entre as equipes por meio do AM.

$\mathrm{E}$, apesar do histórico de alinhamento com os pressupostos da RPB e dos avanços nos processos de desinstitucionalização, as fragilidades na organização da rede de saúde mental na cidade do Recife podem ser agravadas pela perspectiva de remanicomialização do cuidado, explicitada pela agenda antirreformista, que, desde 2017, vem instituindo mudanças na Política Nacional de Saúde Mental.

Diante da complexidade que envolve a política de saúde mental, sugere-se o desenvolvimento de outros estudos que contemplem a perspectiva dos usuários, dos seus familiares e dos profissionais da APS.

\section{Colaboradoras}

Silva AP (0000-0002-7874-4806)* e Morais HMM (0000-0001-8244-6601)* contribuíram igualmente para a concepção, o planejamento, a análise e interpretação dos dados, a elaboração do rascunho, revisão crítica do conteúdo e aprovação da versão final do manuscrito. Albuquerque MSV (0000-0002-1520-700X)* e Guimarães MBL (0000-0001-8554-600X)* contribuíram para análise e a interpretação dos dados, elaboração do rascunho, revisão crítica do conteúdo e aprovação da versão final do manuscrito. Lyra TM (0000-00023600-7250)* contribuiu para a interpretação dos dados, elaboração do rascunho, revisão crítica do conteúdo e aprovação da versão final do manuscrito. 


\section{Referências}

1. Amarante P, Nunes MO. A reforma psiquiátrica no SUS e a luta por uma sociedade sem manicômios. Ciênc. Saúde Colet. 2018; 23(6):2067-2074.

2. Guimarães TAA, Rosa LCS. A remanicomialização do cuidado em saúde mental no Brasil no período de 2010-2019: análise de uma conjuntura antirreformista. Soc. Quest. 2019; (44):111-138.

3. Onocko-Campos RT, Menezes CE, Saraceno AB, et al. Atuação dos Centros de Atenção Psicossocial em quatro centros urbanos no Brasil. Rev. Panam. Salud Publica. 2018; (42):el13.

4 Brasil. Ministério da Saúde. Portaria nº 3.088, de 23 de dezembro de 2011. Institui a Rede de Atenção Psicossocial para pessoas com sofrimento ou transtorno mental e com necessidades decorrentes do uso de crack, álcool e outras drogas, no âmbito do Sistema Único de Saúde. Diário Oficial da União. 26 Dez 2011.

5. Albuquerque MV, Viana ALD. Perspectivas de região e redes na política de saúde brasileira. Saúde debate. 2015; 39(esp):28-38.

6. Magalhães Júnior HM. Redes de Atenção à Saúde: rumo à integralidade. Divulg. Saúde Debate. 2014; (52):5-37.

7. Oliveira RR, Elias PEM. Conceitos de regulação em saúde no Brasil. Rev. Saúde Pública. 2012; 46(3):571576.

8. Vilarins GCM, Shimizu HE, Gutierrez MMU. A regulação em saúde: aspectos conceituais e operacionais. Saúde debate. 2012; 36(95):640-647.

9. Yin R. Estudo de caso: Planejamento e métodos. 5. ed. Porto Alegre: Bookman; 2015.

10. Kvale S. Interviews: an introduction to qualitative research interviewing. London: Sage; 1996.
11. Albuquerque MSV, Costa AM, Lima LP, et al. Equidade e inclusão de grupos sociais na política de saúde: o caso do Recife, Brasil. Rev. Bras. Saúde Mater. Infant. [internet]. 2011 [acesso em 2019 dez 3]; 11(1):73-82. Disponível em: https://doi.org/10.1590/ S1519-38292011000100008.

12. Maia AIS. A política de saúde mental em Recife: caminhos da desinstitucionalização. [dissertação]. Recife: Universidade Federal de Pernambuco; 2007. 112 p.

13. Brasil. Ministério da Saúde. Saúde Mental em Dados $\mathrm{n}^{\mathrm{o}} 12$ : Informativo eletrônico de dados sobre a Política Nacional de Saúde Mental. [internet] Brasília, DF: Ministério da Saúde; 2015. [acesso em 20 maio 2015]. Disponível em: https://www.mhinnovation.net/sites/default/files/downloads/innovation/reports/ Report_12-edicao-do-Saude-Mental-em-Dados.pdf.

14. Gonçalves VM, Candiago RH, Saraiva SS, et al. A falácia da adequação da cobertura dos Centros de Atenção Psicossocial no estado do Rio Grande do Sul. Rev. psiquiatr. Rio Gd. Sul. 2010; 32(1):16-18.

15. Recife. Secretaria Executiva de Atenção à Saúde. Projeto de fortalecimento da rede de atenção psicossocial (Raps). Recife: Secretaria Executiva de Atenção à Saúde; 2014.

16. Recife. Diretoria Executiva de Vigilância à Saúde. População do Recife: censo demográfico 2010 e projeções 2010 a 2018. Recife: Secretaria de Saúde do Recife; $2019.37 \mathrm{p}$.

17. Pinho ES, Souza ACS, Esperidião E. Processos de trabalho dos profissionais dos Centros de Atenção Psicossocial: revisão integrativa. Ciênc. Saúde Colet. 2018; 23(1):141-152.

18. Costa PHA, Colugnati FAB, Ronzani TM. Avaliação de serviços em saúde mental no Brasil: revisão sistemática da literatura. Ciênc. Saúde Colet. 2015; 20(10):3243-3253. 
19. Brasil. Ministério da Saúde. Saúde mental no SUS: os centros de atenção psicossocial. Ministério da Saúde: Brasília, DF; 2004. (Série F: Comunicação e Educação em Saúde).

20. Mendes EV. Comentários sobre as Redes de Atenção à Saúde no SUS. Divulg. Saúde Debate. 2014; (52):3849.

21. Schneider ARS. Mental health care network: the interaction importance between the primary. Ciênc. Saúde. 2010; 2(2):78-84.

22 Furtado JP, Oda WY, Borysow IC, et al. A concepção de território na Saúde Mental. Cad. Saúde Pública. 2016; 32(9):e00059116.

23. Assis JT, Barreiros CA, Jacinto ABM, et al. Política de saúde mental no novo contexto do Sistema de Saúde: regiões e redes. Divulg. Saúde Debate. 2014; (52):88-113.

24. Almeida PF, Giovanella L, Mendonça MHM, et al. Desafios à coordenação dos cuidados em saúde: estratégias de integração entre níveis assistenciais em grandes centros urbanos. Cad. Saúde Pública. 2010; 26(2):286-298.

25. Brasil. Ministério da Saúde. Cobertura da Estratégia da Saúde da Família [internet]. Brasília, DF: Ministério da Saúde; 2017. [acesso em 2017 maio 15]. Disponível em: https://egestorab.saude.gov.br/paginas/ acessoPublico/relatorios/relHistoricoCoberturaAB. xhtml.

26. Gryschek G, Pinto AAM. Saúde Mental: como as equipes de Saúde da Família podem integrar esse cuidado na Atenção Básica? Ciênc. Saúde Colet. 2015; 20(10):3255-3262.

27. Bonfim IG, Bastos ENE, Góis CWL, et al. Apoio matricial em saúde mental na atenção primária à saúde: uma análise da produção científica e documental. Interface (Botucatu). 2013; 17(45):287-300.

28. Melo TMAG. Memórias sobre a política de saúde mental em Recife: entre passos, descompasso e con- trovérsias [tese]. Recife: Universidade Federal de Pernambuco; 2017. 253 p.

29. Figueiroa MS. Avaliação da implantação do apoio matricial do CAPS AD na cidade do Recife [dissertação]. Recife: Instituto de Medicina Integral Professor Fernando Figueira; 2012.118 p.

30. Gazignato ECS, Silva CRC. Saúde mental na atenção básica: o trabalho em rede e o matriciamento em saúde mental na Estratégia de Saúde da Família. Saúde debate. 2014; 38(101):296-304.

31 Arce VAR, Sousa MF, Lima MG. A práxis da Saúde Mental no âmbito da Estratégia Saúde da Família: contribuições para a construção de um cuidado integrado. Physis. 2011; 21(2):541-60.

32. Severo AK, Dimenstein M. Rede e intersetorialidade na atenção psicossocial: contextualizando o papel do ambulatório de saúde mental. Psicol., Ciênc. Prof. 2011; 31(3):640-655.

33. Damous I, Erlich H. O ambulatório de saúde mental na rede de atenção psicossocial: reflexões sobre a clínica e a expansão das políticas de atenção primária. Physis. 2017; 27(4):911-932.

34. Ferrazza DA, Luzio CA, Rocha LC, et al. A banalização da prescrição de psicofármacos em um ambulatório de saúde mental. Paidéia. 2010; 20(47):381-390.

35. Garla CC, Furegato ARF, Santos JF. Profissionais de Ambulatórios de Saúde Mental: perfil, práticas e opiniões sobre as políticas. Cad. Bras. Saúde Ment. 2010; 2(4-5):74-93.

36. Santos YF, Oliveira IMFF, Yamamoto OH. O ambulatório da saúde mental no contexto da reforma psiquiátrica em natal RN. Psicol. Argum. 2009; 27(59):313322.

37. Sousa FOS, Medeiros KR, Gurgel Júnior GD, et al. Do normativo à realidade do Sistema Único de Saúde: revelando barreiras de acesso na rede de cuidados assistenciais. Ciênc. Saúde Colet. 2014; 19(4):1283-1293. 
38. Tesser CD, Poli Neto P. Atenção especializada ambulatorial no Sistema Único de Saúde: para superar um vazio. Ciênc. Saúde Colet. 2017; 22(3):941-951.

39. Chazan LF, Fortes S, Camargo Jr. KR, et al. O apoio matricial na Atenção Primária em Saúde no município do Rio de Janeiro: uma percepção dos matriciadores com foco na Saúde Mental. Physis. 2019; 29(2): 290212.
40. Baduy RS, Feuerwerker LCM, Zucoli M, et al. A regulação assistencial e a produção do cuidado: um arranjo potente para qualificar a atenção. Cad. Saúde Pública. 2011; 27(2):295-304.

Recebido em 26/02/2020

Aprovado em 18/11/2020

Conflito de interesses: inexistente

Suporte financeiro: não houve 